\title{
Physical Therapy in the Management of Recent Paralysis in Leprosy
}

\author{
M. A. FURNESS, M.c.s.P. \\ Physiotherapist, Schieffelin Leprosy Research Sanatorium, B.O., Karigiri, \\ via Katpadi, N.A. Dist., S. India
}

A common feature of recent paralysis in leprosy is the presence of infiltration by inflammatory exudate and oedema in the affected nerve which causes ischaemia in the nerve. ${ }^{1}$ Partial ischaemia causes a neuropraxia or impairment of nerve conduction without Wallerian degeneration. When blood flow returns, conductivity of the nerve is restored to normal. If the ischaemia progresses to complete loss of blood supply or lasts for a long time, then the nerve is destroyed and the paralysis becomes irreversible. Loss of conductivity of a nerve nearly always precedes nerve destruction. It has been observed that this stage may last for a short while or extend over a period of months and be followed by recovery.

This paper outlines the principles of physical therapy used in recently paralysed muscles in leprosy and explains the methods employed to preserve the physiological properties of the muscles and prevent atrophy and deformity during the stage of paralysis, and the techniques used to re-educate and strengthen muscles during the recovery phase. It must be emphasised that physical therapy in these cases is supple. mentary to adequate and proper medical ca: under the supervision of a physician.

\section{MATERIAL}

This study was conducted on a group of 36 patients in whom the duration of paralysis ranged from 4 days to 6 mo:ths: 12 presented with lateral popliteal paralysis; 7 with radial paralysis; and 17 with ti.ulal paralysis. Their duration of treatment ranged from 5 weeks to 5 months. All patients continued to be under medical treatment during their period of physical therapy. At Schieffelin Leprosy Research Sana- torium specific anti-leprosy treatment is stopped in all patients who are found to have recently developed neurological deficit and appropriate anti-inflammatory drug is introduced, the choice of which depends upon the clinical condition of the patient.

\section{ASSESSMENTS}

The following assessments were made on each patient and repeated at regular intervals.

1. The Manual Muscle Test using the international 1 to 5 scale was recorded periodically on a form to facilitate quick and accurate reviews.

2. Sensory Test. Both tactile and pain sensations were tested and recorded on maps of the extremities at regular intervals.

\section{PHYSICAL THERAPY}

Since neuropraxia in leprosy may last for a long time, it is necessary to prevent atrophy and deformity and prolonged overstretching of the paralysed muscles during the period the nerve is not functioning. Attempts must be made to initiate a response from these muscles as early as possible so that function can be quickly restored.

The paralysis was therefore treated in 2 phases:-

(a) the expectant phase in which signs of recovery were not yet present; and

(b) the re-educative phase in which recovery had already begun.

\section{PRINCIPLES OF PHYSICAL THERAPY}

\section{(a) In the Expectant Phase}

1. To prevent contractures due to muscle imbalance and to retain mobility of the joints. 


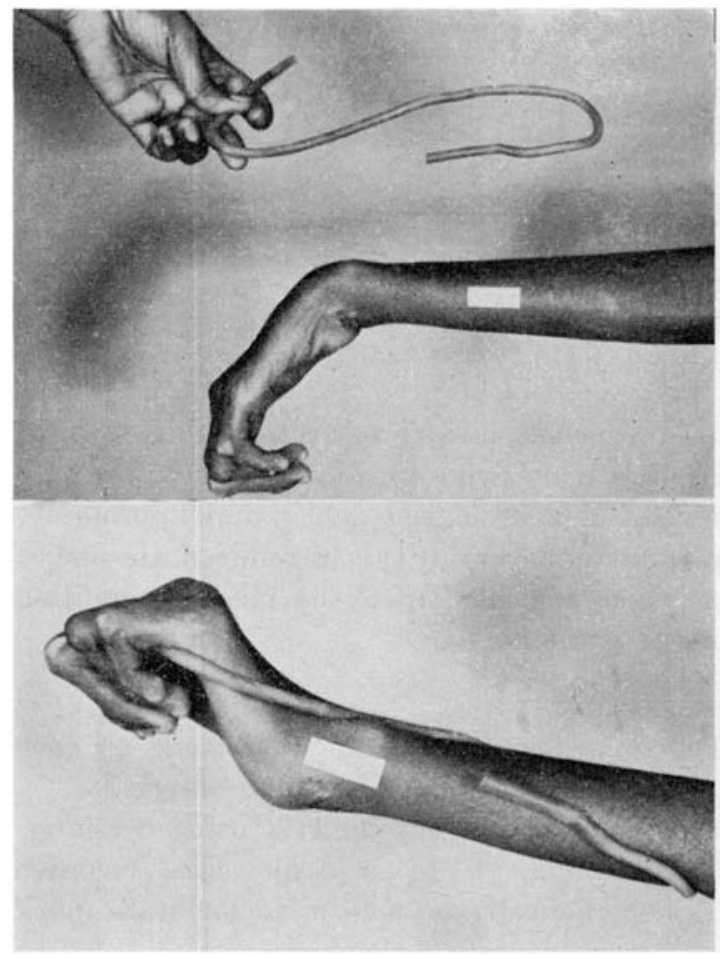

FIG. 1

Simple ' $S$ '-shaped splint with soft rubber covering for wrist support and allowing free finger movements.

2. To prevent atrophy of the muscles.

3. To prevent prolonged overstretching of the paralysed muscle and to retain the denervated muscle in an adequate nutritional state until re-innervation.

The methods to implement these principles were:-

(i) Passive Movements. These movement were used to prevent contractures that may arise in joints due to muscle imbalance and disuse. In radial paralysis passive movements were used daily for the joints of the wrist, fingers and the thumb. In lateral popliteal paralysis the ankle joint and the small joints of the foot were put through full range of movements.

(ii) Massage. Light effleurage and finger kneadings were used for muscles of the face to increase circulation in the facial muscles, and to maintain them in good

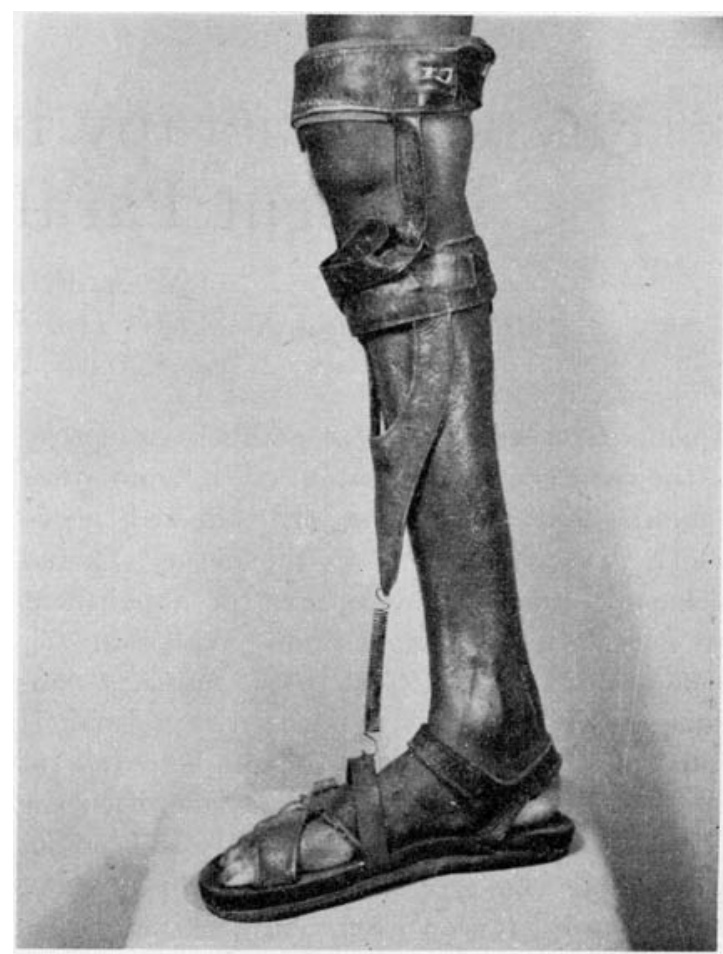

FIG. 2

Standard foot-drop spring. The spring and buckles are adjusted to prevent excessive dorsiflexion.

nutritional state until recovery of function occurred.

(iii) Electrical Stimulation. When there is a neuropraxia of a motor nerve, impulses from the brain fail to pass the site of the lesion to reach the muscles supplied by the nerve. Contractions of the muscles were therefore obtained by a faradic stimulus applied below the site of the lesion. Treatments were given twice a day. Since these contractions closely simulate voluntary contractions they help to preserve a sense of movement and prevent atrophy that may be caused by disuse of the muscles.

(iv) Splinting. Splinting was used to prevent prolonged overstretching of the paralysed muscle which would damage its physiological properties of elasticity, extensibility and contractility and retard re- 
covery. Splints were also employed to prevent adaptive shortening of the antagonist muscles and to allow functional use of the limb. Patients with a radial paralysis were provided with an 'S' shaped splint which maintained the wrist in dorsiflexion while allowing free movements of the fingers (Fig. 1). Lateral popliteal paralysis was treated with a dorsiflexion shoe spring during the day (Fig. 2), which allowed functional use of the limb for walking, and a posterior slab to hold the foot at $90^{\circ}$ during the night. Sagging facial muscles were strapped with adhesive plaster to prevent prolonged overstretching and were removed only during exercise periods.

\section{(b) In the Re-educative Phase}

1. To develop motor awareness and voluntary response.

2. To strengthen progressively the affected muscles by resisted exercises which were specific for the paralysed group.

3. To restore full function of the affected muscles by skilled, co-ordinated movements.

Since contraction is the only means by which muscles regain their normal function it is essential to obtain a response as soon as possible from the muscles affected by paralysis. Besides, the longer the muscles remain inactive the greater will be the tendency for disuse atrophy. Therefore, when the acute phase of the lesion had subsided, reactivation of the motor unit was undertaken and proved possible except where there had been destruction of axons. In patients in whom the demands made on the neuromuscular system by voluntary effort were insufficient to elicit the maximal responses required for contraction and rapid re-development of muscles, methods designed to stimulate or augment the demand for activity were instituted:-

(i) Facilitation techniques. The techniques of proprioceptive neuromuscular facilitation ${ }^{1}$ which rely mainly on the use of the stretch stimulus to initiate tension within paralysed muscles through the stretch receptors and muscle spindle mechanism, were used to initiate contraction in recovering muscles. Resistance and stretch were applied manually to muscles working to perform patterns of movement in patients with radial and lateral popliteal paralysis. The stretch stimulus was also applied to facial muscles to obtain contraction of these muscles.

(ii) Exercises. Once the power of contraction was gained active exercises were used eliminating gravity, and were progressed to exercises against gravity. When muscle fatigue was noticed in weak muscles, periods of rest were given during the performance of the exercise. When a muscle was able to perform a movement against gravity and was graded 3, exercises against resistance were commenced to hypertrophy the muscle. At this stage support, passive movements, assisted active movements and artificial means of maintaining circulation were gradually discontinued.

Resisted exercises for radial, lateral popliteal and facial paralysis were given manually. Weights were used at a later stage as a means of resistance in lateral popliteal paralysis.

Co-ordination was improved by repetition of an exercise so that exercises and activities which at one time required concentration and much effort became, with practice, more or less automatic in character and function of the limb was improved.

Occupational therapy played an important part in the management of recovering nerve lesions in leprosy. Exercises were made more interesting and dexterity was improved in the performance of movements. Work in the department was directed towards the skills necessary for the patient's previous occupation.

\section{RESULTS}

A total number of 36 patients were treated: 7 with radial paralysis; 12 with lateral popliteal paralysis; and 17 with facial paralysis. All 
5 lepromatous patients with paralysis showed recovery. In the tuberculoid type 12 out of a total of 16 , and in the borderline type 11 out of a total of 15, recovered. The total percentage of recovery was $78 \%$.

The average duration of paralysis among those who failed to recover was 5 months. Patients whose average duration of paralysis was 2.75 months, recovered.

This series is too small to permit valid generalisation, though it would substantiate the clinical impression that the chances of recovery in paralyses in leprosy are related to the type of leprosy and the duration of paralysis before institution of adequate and supervised medical treatment and physical therapy.

TABLE 1

Recovery according to Classification

\begin{tabular}{lcc}
\hline Classification & $\begin{array}{c}\text { Numbers } \\
\text { Treated }\end{array}$ & $\begin{array}{c}\text { Numbers } \\
\text { Recovered }\end{array}$ \\
\hline Lepromatous & 5 & 5 \\
Tuberculoid & 16 & 12 \\
Borderline & 15 & 11 \\
\hline TOTAL & 36 & $28(78 \%)$ \\
\hline
\end{tabular}

TABLE 2

Duration of Paralysis and Duration of Treatment

\begin{tabular}{lcc}
\hline & $\begin{array}{c}\text { Patients } \\
\text { Recovered }\end{array}$ & $\begin{array}{c}\text { Patients } \\
\text { Not Recovered }\end{array}$ \\
\hline $\begin{array}{c}\text { Average Duration } \\
\text { of Paralysis }\end{array}$ & 2.75 months & 5 months \\
\hline $\begin{array}{l}\text { Average Duration } \\
\text { of Treatment }\end{array}$ & 2.25 months & 4 months \\
\hline
\end{tabular}

\section{SUMMARY}

1. The patho-physiology of nerve lesions in leprosy is briefly described.

2. The principles and methods of physical therapy during the expectant phase and the re-educative phase are described and discussed.

3. The clinical results in 36 patients with recent onset of peripheral nerve paralysis (radial 7, lateral popliteal 12, facial 17) treated at the Schieffelin Leprosy Research Sanatorium are presented.

\section{ACKNOWLEDGEMENTS}

I would like to thank I)r. C. K. Job, Superintendent, for permission to publish this paper; Dr. A. B. A. Karat, Consultant Physician, and Mrs. A. Karat, Consultant Surgeon, for the opportunity to study patients under their care, and for helpful criticism and encouragement; Mr. C. Dorairaj for help with the photographs; and Mrs. L. Furness for secretarial assistance.

\section{BIBLIOGRAPHY}

1. BRAND, PAUL w. 'Temperature Variation and Leprosy Deformity.' Int. .J. Lepr., 27, 1, 1959.

2. кават, н. and кnотт, м. 'Proprioceptive Facilitation Technics for Treatment of Paralysis.' Phys. Ther. Rev., 33, 58, 1963. 